\title{
New Insights into Ligand-Receptor Pairing and Coevolution of Relaxin Family Peptides and Their Receptors in Teleosts
}

\author{
Sara Good, ${ }^{1}$ Sergey Yegorov, ${ }^{1}$ Joran Martijn, ${ }^{2}$ Jens Franck, ${ }^{1}$ and Jan Bogerd ${ }^{2}$ \\ ${ }^{1}$ Department of Biology, University of Winnipeg, Winnipeg, MB, Canada R3B 2E9 \\ ${ }^{2}$ Department of Biology, Faculty of Science, University of Utrecht, $3584 \mathrm{CH}$ Utrecht, The Netherlands
}

Correspondence should be addressed to Sara Good, s.good@uwinnipeg.ca and Jan Bogerd, j.bogerd@uu.nl

Received 30 March 2012; Revised 7 June 2012; Accepted 15 June 2012

Academic Editor: Frédéric Brunet

Copyright ( 2012 Sara Good et al. This is an open access article distributed under the Creative Commons Attribution License, which permits unrestricted use, distribution, and reproduction in any medium, provided the original work is properly cited.

\begin{abstract}
Relaxin-like peptides (RLN/INSL) play diverse roles in reproductive and neuroendocrine processes in placental mammals and are functionally associated with two distinct types of receptors (RXFP) for each respective function. The diversification of RLN/INSL and RXFP gene families in vertebrates was predominantly driven by whole genome duplications ( $2 \mathrm{R}$ and $3 \mathrm{R}$ ). Teleosts preferentially retained duplicates of genes putatively involved in neuroendocrine regulation, harboring a total of 10-11 receptors and 6 ligand genes, while most mammals have equal numbers of ligands and receptors. To date, the ligand-receptor relationships of teleost $\mathrm{R} \ln /$ Insl peptides and their receptors have largely remained unexplored. Here, we use selection analyses based on sequence data from 5 teleosts and qPCR expression data from zebrafish to explore possible ligand-receptor pairings in teleosts. We find support for the hypothesis that, with the exception of RLN, which has undergone strong positive selection in mammalian lineages, the ligand and receptor genes shared between mammals and teleosts appear to have similar pairings. On the other hand, the teleostspecific receptors show evidence of subfunctionalization. Overall, this study underscores the complexity of RLN/INSL and RXFP ligand-receptor interactions in teleosts and establishes theoretical background for further experimental work in nonmammals.
\end{abstract}

\section{Introduction}

Relaxin-like peptides are members of the insulin superfamily and, like insulin and insulin-like growth factors (IGF), are small peptides ( $\sim 60$ amino acids) that share a common twodomain structure (A and B domains) in their mature form [1]. Functionally, however, relaxin family peptides are different from insulin and IGF: they bind to unrelated receptors and play diverse roles in reproduction and neuroendocrine regulation as opposed to carbohydrate/fat metabolism and growth. Four relaxin family peptide-encoding genes ( $R L N$, RLN3, INSL3, and INSL5) originated early in vertebrate history and are shared by most vertebrates [2]. The receptors for the RLN/INSL peptides belong to two distinct groups of $G$ protein-coupled receptors (GPCR), collectively named the relaxin family peptide receptors (RXFP) [3].

In mammals, there are four known receptors, RXFP1-4, associated with the four relaxin family ligands. RXFP1 and RXFP2 are evolutionarily related to glycoprotein hormone receptors (e.g., luteinizing and follicle-stimulating hormone receptors), containing a large extracellular domain made up of ten leucine-rich repeats (LRR) and a low-density lipoprotein receptor type A (LDLa) module; they are the cognate receptors for the ligands RLN and INSL3 in humans, both of which primarily have reproductive actions [3]. On the other hand, RXFP3 and RXFP4 are classic type I peptide GPCRs with short N-terminal domains; they are evolutionarily related to somatostatin and angiotensin receptors and, in humans, are the cognate receptors for RLN3 and INSL5, both of which are associated with neuroendocrine signaling [3].

The two hormones with reproductive functions in mammals, RLN and INSL3, are the best understood. The hormone RLN is well known for its role in parturition, where it softens connective tissues of the reproductive tract via tissue remodeling and prepares the mammary glands for lactation, but it has numerous other physiological actions as well [1]; its receptor (RXFP1) also exhibits a wide distribution suggesting endocrine action in mammals [7] (Table S1, see supplementary materials available online at 
doi: 10.1155/2012/310278). In teleosts, the peptide sequence of Rln is highly similar to that of Rln3 [8]; although its function remains unknown, the $r \ln$ gene exhibits substantial overlap in expression with $r \ln 3$, both being highly expressed in brain, although teleost $r \ln$ is also significantly expressed in gonads [9]. While mammalian and teleost RLNs differ somewhat in their expression patterns, INSL3 has a more similar expression pattern in the two lineages; it is highly expressed in Leydig cells in both mammals [10] and teleosts [8], and at lower levels in other tissues (see Table S1). In mammals, the receptor for INSL3, RXFP2, is also highly expressed in testes suggesting paracrine action [6], but lower levels of RXFP2 expression are observed in a wide array of tissues [7]. The receptor has been, until now, unstudied in teleosts.

The peptides RLN3 and INSL5 exert their influence primarily through the hypothalamic-pituitary-gonadal (HPG) axis $[11,12]$. RLN3 is the most conserved member of the family; it is predominantly expressed in the nucleus incertus (NI) in mammalian brain [13] and its homologous region in teleosts [14]. Ascending RLN3-producing projections from the NI innervate a broad range of RXFP3-expressing regions of the forebrain in mammals, including the hypothalamus and it is implicated in the acute stress response and regulation of food intake $[12,15]$. Collectively, these lines of evidence suggest that RLN3 acts through the HPG axis and may play a dual role linking nutritional status to reproductive function [12]. Lastly, INSL5 is the least well understood member of the family, but in humans its primary sites of expression are rectum, colon, and uterus $[16,17]$ (see Table S1). The receptor for INSL5 in mammals, RXFP4, has a wide distribution being found in colon, placenta, testis, thymus, prostate, kidney, and brain in human [18], strongly suggesting endocrine action.

Despite the evolutionary distance separating RXFP1/2 and RXFP3/4-type receptors, experimental studies have shown that some RLN/INSL peptides can bind additional (secondary) receptors at lower affinity [5]. For example, in addition to RXFP3, RLN3 can bind to and activate RXFP1 and RXFP4, RLN can bind to RXFP2 in addition to RXFP1 [19], and INSL5 can bind to (but activate only weakly) RXFP3 in addition to its primary receptor RXFP4. Such "primary" and "secondary" ligand-receptor interactions have been demonstrated for human RLN/INSLRXFP pairs, but analogous pairings in other vertebrates, such as teleosts, in which relaxin family peptide-receptor signaling and diversification have taken an evolutionary pathway distinct from that in mammals [2], remain to be established.

Recent evolutionary analyses revealed that vertebrate RLN/INSL genes and their receptors primarily diversified through the two rounds (2R) of whole genome duplication (WGD) that occurred in early vertebrate evolution and, in teleosts, during the teleost fish-specific WGD (3R) (Figure 1). To summarize, mammals retained 4 ligand and 4 receptor genes following $2 \mathrm{R}$, while teleosts have 10 (most teleosts) or 11 (zebrafish) receptor and 6 ligand genes following 3R (Figure 1) and after-3R local duplications (Figure 2, Table S2). Many of the genes retained in duplicate in fish $(r \ln 3-$, insl5-, and $r x f p 3$-type genes) are hypothetically involved in neuroendocrine regulation (Figure 3). But due to a lack of understanding of the evolutionary history of $r \ln / i n s l$ and $r \times f p$ genes in teleosts, the ligand-receptor pairings in teleosts are virtually unknown.

One of the interesting aspects of the evolution of RLN/INSL peptides is how a set of relatively closely related ligands signals via two unrelated types of receptors. Yegorov and Good [2] hypothesized that this dual-functioning arose in the ancestral pre-2R RLN/INSL peptide that had roles in both reproductive (via RXFP1/2-receptor) and neuroendocrine (via RXFP3/4) regulations in primitive vertebrates (Figure 3). As a result of the WGDs, the ancestral tripartite system gave rise to two distinct parties of RLN/INSLRXFP ligand-receptor pairs (Figure 3). Curiously, it can be observed that, with the exception of the RXFP1 receptor and its ligand RLN, each of the duplication events resulted in a single ligand that potentially could function with two related receptors (Figure 3). In most mammals, this tripartite model became reduced to a $1: 1$ relationship for ligands and receptors after the divergence of tetrapods from the gnathostome ancestor (as described above), but in teleosts, there are multiple receptors for some ligands, which may have occurred through receptor subfunctionalization (Figure 3). Based on the evolutionary history of duplication, and the ligand-receptor pairings in mammals, we developed hypotheses concerning which ligand-receptor pairings we expect in teleosts (Figure 4). The primary goal of this paper is to test our hypotheses about the Rln/Insl-Rxfp ligand-receptor pairs in teleosts using selection analyses and experimental qPCR data from zebrafish.

\section{Results}

2.1. Selection Analyses. We performed two kinds of molecular evolutionary analyses to (1) hypothesize which ligandreceptor pairings may occur in teleosts and (2) examine differences in selection among mammalian and teleost genes.

(1) Previous studies have used the correlation of evolutionary distances between putative ligand-receptor pairs as evidence of cofunctioning $[20,21]$. Here, we employed a similar correlation approach, but rather than comparing the mean evolutionary distances among gene pairs, we compared the proportion of sites under different forms of selection (purifying, neutral, or positive) in pairs of teleost genes to the "primary" ligand-receptor pairs known to exist in mammals, $r \ln$-rxfp1, insl3-rxfp2, and $r \ln 3-r x f p 3-1$. If the genes coding for the ligands and receptors coevolve, we expect a correlation in the rates and types of selection on ligand-receptor pairs. This would correspond to values falling along the $(0,0: 1,1)$ plane of the XY-plot. On the other hand, a similar $[\mathrm{X}, \mathrm{Y}]$-value for the same ligand-receptor pair in mammals and teleosts would suggest that the pair plays a similar role in the two lineages.

(2) We tested for evidence of (a) codon-specific positive selection in mammalian and teleost ligand and receptor genes and (b) codon-specific positive selection in mammalian versus teleost genes using the branch-site model of 


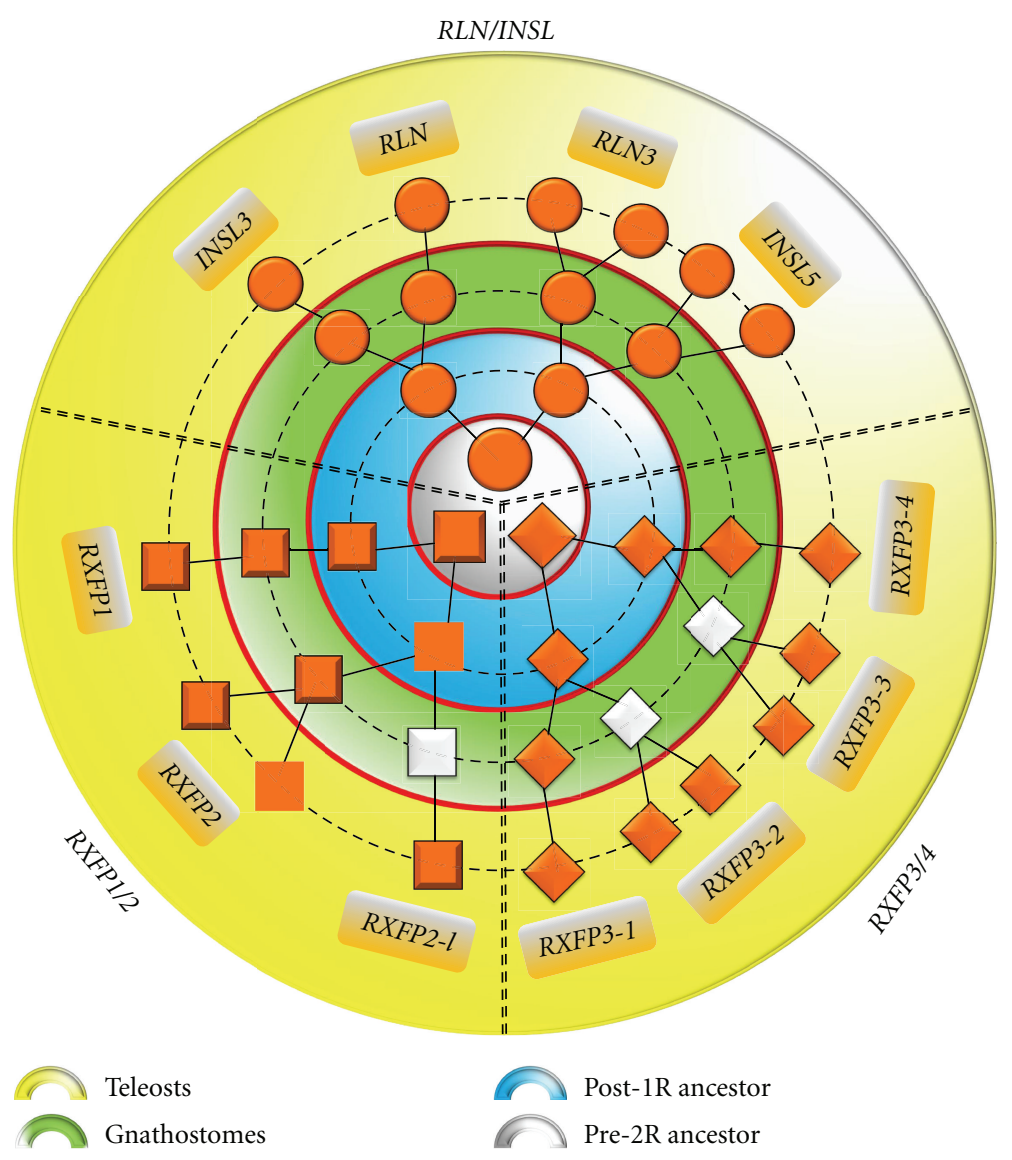

FIgure 1: The role of Whole Genome Duplications (WGD) in the expansion of the RLN/INSL and RXFP genes in vertebrates. The three gene families (one ligand (circles) and two receptor (squares) families) arose as a result of WGDs (1R, 2R, and 3R) from three ancestral genes. RLN/INSL peptides: following $1 \mathrm{R}$, there were two RLN/INSL-like genes, following $2 \mathrm{R}$ one of these gave rise to RLN3 and INSL5, and the other to RLN and INSL3. After the teleost fish-specific WGD (3R), the duplicates of $r \ln 3$ and insl5 were retained bringing the total number of $r \ln /$ insl genes in teleosts to 6. RXFP3/4 receptors: four RXFP3/4-type receptor genes were generated from a single-ancestral gene during $2 \mathrm{R}$; all four of these genes were retained in teleosts, but in tetrapods, only two receptors, RXFP3 (termed RXFP3-1) and RXFP4 (RXFP3-4), were retained. After $3 \mathrm{R}$, the duplicates of $r x f p 3-2$ and $r x f p 3-3$ were retained. RXFP1/2 receptors: most vertebrates have only a single copy of RXFP1 and RXFP2, a few (opossum, frog, reptiles, and zebrafish) have RXFP2-like. Layers coloured in four distinct colors indicate ancestral stages (legend below); WGDs are depicted as red lines surrounding these ancestral stages. White shapes indicate genes lost in most $(R X F P 2-l$, RXFP3-3) or all (RXFP3-2) tetrapod lineages. RXFP2-l= RXFP2-like. Based on Yegorov and Good [2].

positive selection. While the first analysis (a) tests whether specific codons have been positively selected within lineages, the second (b) looks for evidence that codons have been differentially selected in mammalian versus teleost lineages.

(1) Evidence for Ligand-Receptor Coevolution for Mammalian and Teleost Orthologs. Between 70 and $93 \%$ of the sites across all genes, and in both mammals and teleosts, have been subject to purifying selection (Figure 5(a)). Additionally, the extent of purifying selection was symmetric for the ligandreceptor pairs $r \ln 3-r x f p 3$ and insl3-rxfp2 suggesting close coevolution, while for the remaining two pairs, $r \ln -r x f p 1$ and insl5-rxfp4, the proportion of sites under purifying selection was higher for the receptor genes (between 0.7 and 0.92 ) than for the ligands (ranging from 0.4-0.95), suggesting a more diffuse coevolution (or no coevolution), and more relaxed evolution on the ligand.

On the other hand, there are significantly fewer sites which are evolving neutrally (Figure 5(b)) or are subject to positive selection (Figure 5(c)). For the receptor genes, from 3 to $20 \%$ of the sites were found to be evolving neutrally (Figure 5(b)), and from 2 to $13 \%$ were subject to positive selection; $r x f p 3$ exhibits the fewest neutral or positively selected sites, $r x f p 4$ has the highest proportion of sites under neutral evolution and $r x f p 2$ exhibits the highest proportion of sites under positive selection. Largely due to the anomalous nature of asymmetric selection on the $r l n-r x f p 1$ ligand-receptor system in mammals, the extent of neutral and positive selection among ligand genes varied widely between mammals and teleosts, primarily because teleost $r \ln$ was found to have a large number of sites evolving neutrally, whereas mammalian $R L N$ has a large proportion 




Figure 2: Post 3R gene loss and gain in five teleost fish species. Following 3R, teleosts start with a gene set composed of 10 receptors and 6 ligands. Prior to divergence of zebrafish, $r x p p 3-3 a$ is locally duplicated, generating tandem genes $r x f p 3-3 a 1$ and $r x f p 3-3 a 2$. Zebrafish retains most of the genes, except $r x f p 4$, but gains an additional copy of the $r x f p 3-3$ gene, $r x f p 3-3 a 3$, through SSD. Other teleosts lose $r x f p 2-l i k e$ and also the 3R-duplicate $r x f p 2 b$. SSD: small-scale (local) duplication. 3R: fish-specific WGD. Data from Yegorov and Good [2]. Phylogeny and classification of fish adapted from Kinoshita et al. [4].

of sites subject to positive selection (Figures 5(b) and 5(c), resp.).

The Selection Analysis Supports Our Hypothesis for Many Ligand-Receptor Pairs in Teleosts, but the Receptors for the Two Insl5 Paralogs Remain Unclear. Given the presence of additional ligand and receptor genes in teleosts for which no ortholog was present in mammals, the correlation approach could not be used for the additional ligand-receptor genes in teleosts because there was no reference comparison in mammals and too many possible pairs to consider. Thus, to examine the possible pairings of these additional genes, we simply plotted the proportion of sites subject to each form of selection in teleosts for visual comparison (Figure 6). This revealed that the gene coding for Rln has a higher number of neutrally evolving sites than the gene of its proposed receptor, Rxfp1, although this may be an artifact of the comparison to mammalian RLN. On the other hand, the numbers of selected sites in the genes of the proposed ligand-receptor pairs insl3-rxfp2 (as demonstrated above), $r \ln 3 a-r x f p 3-2 a / r x f p 3-2 b$, and $r \ln 3 b-r x f p 3-1$ were similar, supporting possible cofunctioning, although $r x f p 3-2 a$ shows a higher fraction of positively selected sites than either of the $r \ln 3$ ligand genes. Lastly, however, there was also a poor correlation in the expected selection profile of insl5 compared with its proposed receptor genes: both teleost insl5a and insl5b evolve relatively neutrally but none of their proposed receptors do, with the exception of $r x f p 4$, which has a slightly higher rate of neutral and positive selection. The remaining three $r x f p 3-3$ receptor genes are very conserved (Figure 6). Thus, although teleost insl5 and rxfp4 genes had similar selection profiles to those of mammals (see above), suggesting a conserved function between the two lineages, the other three proposed receptors for the insl5 paralogs (i.e., $r x f p 3-3 a 1, r x f p 3-3 a 2$ and $r x f p 3-3 b$ ) exhibited strong 


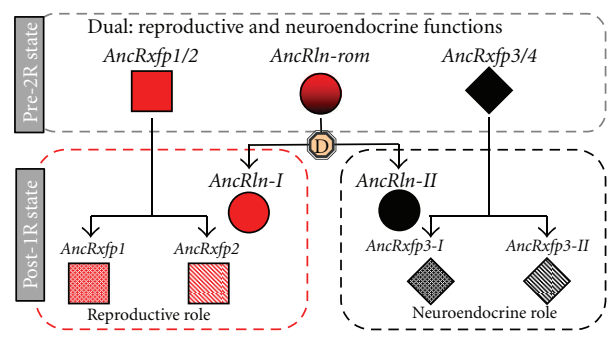

(a)

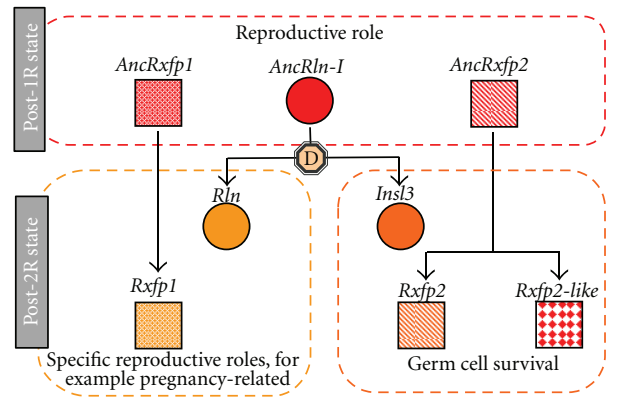

(b)

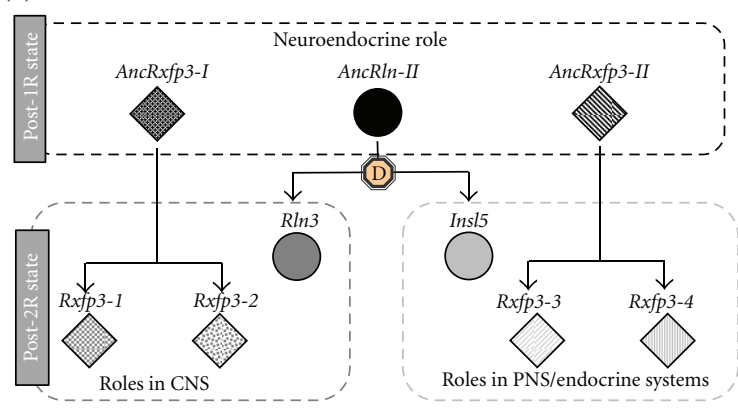

(c)



(d)

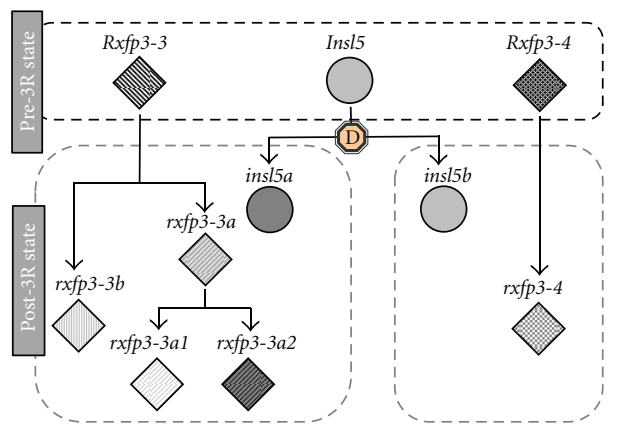

Duplication/divergence

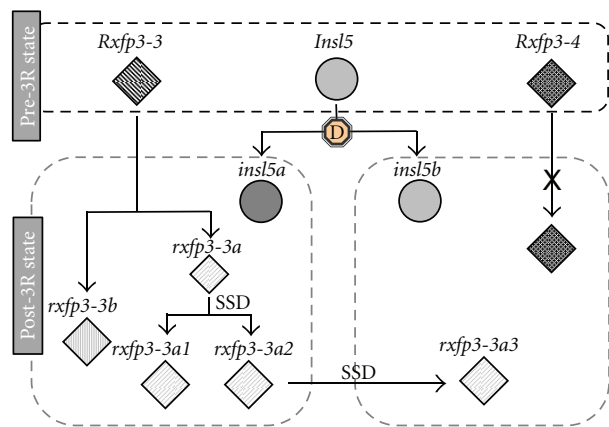

Duplication/divergence

(e)

(f)

Figure 3: The hypothesized functional diversification of the rln/insl and $r x f p$ genes in the gnathostome ancestors (a, b, and c) and in teleosts ( $\mathrm{d}$, e, and f). (a) The pre-1R three-gene system gave rise to two ligand genes and two pairs of receptor genes following 1R. After 1R, both ligands and receptors are structurally and functionally identical, which is favorable for promiscuous ligand-receptor interactions, in combination with selective pressures promoting a division of reproductive and neuroendocrine systems, leading to the establishment of novel ligand-receptor pairs. (b) Duplication and divergence of the $r \ln -r x f p 1$ and insl3-rxfp2 ancestor genes. On the basis of the proposed relatedness of $r x f p 2$-like to $r x f p 2$, we hypothesize that Rxfp-like, at least immediately after $2 \mathrm{R}$, functioned as a receptor for Insl3. (c) Duplication and divergence of the genes ancestral to $r \ln 3$ and insl5 and their rxfp3/4-type receptor genes. Since all tetrapods lost $r \times f p 3-2$ and most of them also lost $r x f p 3-3$, their ligand-receptor pairs lost their ancestral three-component nature and became two-component, that is, Rln3-Rxfp3-1 and Ins15-Rxfp4. (d) Teleosts retained all after-2R rxfp3/4 receptor genes and seem to have experienced further subfunctionalization with the formation of complex ligand-receptor relationships. We hypothesize a functional specialization of the two $r \ln 3$ paralogs to work with $r x f p 3-1$ $(r \ln 3 a)$ and two $r x f p 3-2$ genes. (e) Diversification of $r x f p 3-3$ and $r x f p 3-4$ genes in percomorpha (f) Zebrafish has lost its $r x f p 3-4$ (i.e., $r x f p 4$ ) gene but has an extra copy of $r x f p 3-3 a 3$, which may imply that the receptor of Insl5b is Rxfp3-3a3. Note that in (b) and (c) insl5 paralogs are chosen arbitrarily and the interaction of the peptide with the receptors can be reversed; that is, Insl5a may function with Rxfp3-4 and Insl5b may interact with $\mathrm{Rxfp3}-3$ receptors $\mathrm{SSD}=$ small scale duplication. 


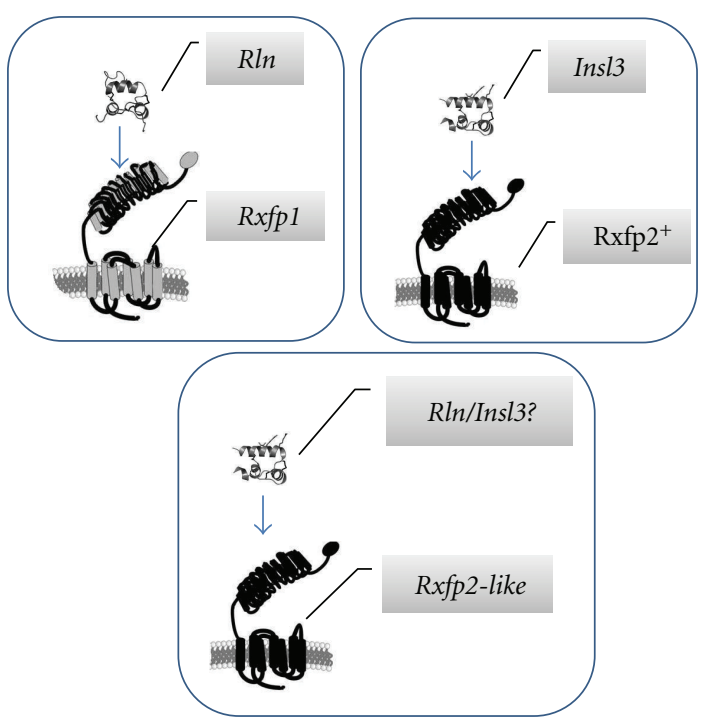

(a)

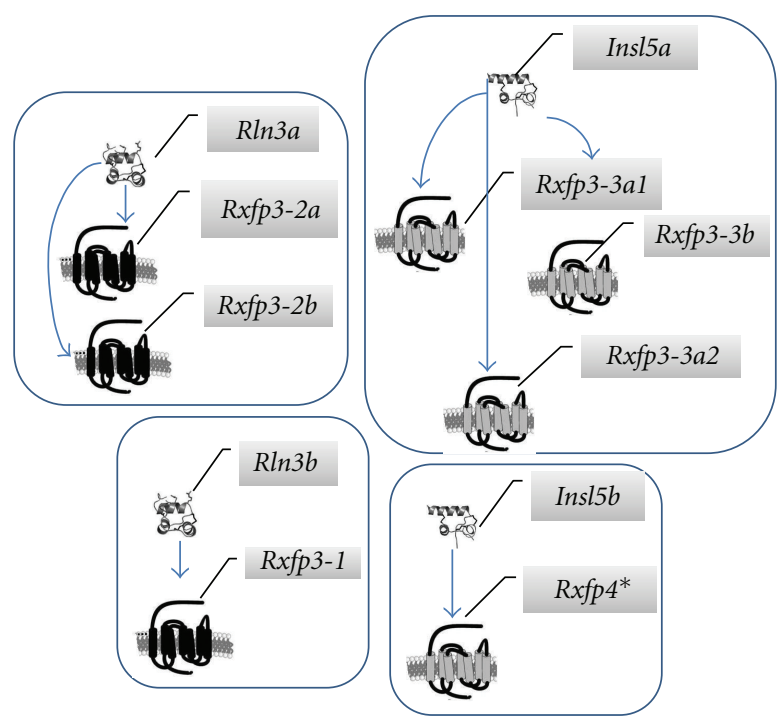

(b)

FIGURE 4: Ligand-receptor pairings of the Rln/Insl peptides and their Rxfp receptors putatively associated with (a) reproductive and (b) neuroendocrine processes in teleosts as hypothesized based on mammalian pairings and on their gene duplication history (see Figures 1 and 3). Few tetrapods (reptiles, frog, and opposum) and zebrafish have been found to possess the receptor rxfp2-like, which is phylogenetically more closely related to $r x f p 2$ than $r x f p 1$, but still of ancient origin [2]. However, the lack of insl3 in the reptiles, that also harbour $r x f p 2$-like (data from [2]), suggests that Rln may be an alternate ligand. + Zebrafish retained two 3R paralogs of $r x f p 2$, $r x f p 2 a$, and $r x f p 2 b$, while the remaining teleosts appear to have lost one copy. *In zebrafish, the $r x f p 4$ gene was lost and possibly replaced by rxfp3-3a3 (see Figures 3 and 4). Images of receptors and peptides adopted with permission from the publisher for Halls et al. [5] and Kong et al. [6].

purifying selection and did not closely parallel the selection profile of either candidate ligands.

(2a) Evidence for Codon-Specific Positive Selection in Mammalian and Teleost Ligand and Receptor Genes. To look for evidence of codon-specific positive selection in mammalian and teleost lineages, we compared models 7 (purifying selection), 8 (positive selection), and 8 a (relaxation of purifying selection) using maximum likelihood-based comparisons [22] in mammals and teleosts. Genes are considered to be under positive selection if the support for model 8 is greater than model 7 , but also model $8 \mathrm{a}$. For genes that exhibited evidence of positive selection, determination of the amino acid sites estimated to be under selection was tested using Bayesian Empirical Bayes (BEB). We found evidence of positive selection for mammalian INSL5 and mammalian $R L N$; however, the hypothesis that the positive selection found in mammalian INSL5 is actually caused by a relaxation of purifying selection (i.e., tested by comparing model 8a versus model 8) could not be rejected. The extent of positive selection on mammalian $R L N$ is extensive; however, in total, 12 amino acid positions were identified as having a BEB probability $>0.9$ that $\omega>1.0$ (i.e., to be under positive selection) and another five had a probability $>0.8$ that $\omega>1.0$ (Table S3). This suggests the presence of strong diversifying selection on mammalian $R L N$. In teleosts, only insl3 showed evidence of having codons subject to positive selection at two sites (Table S3).

There was some, but limited, evidence of positive selection on the receptor genes within mammalian or teleost lineages. Only one codon was found to exhibit strong evidence of positive selection in mammalian $R X F P 1$, and two for RXFP2, while three codons showed evidence of positive selection in fish $r x f p 2$, but the latter hypothesis was more likely attributed to a relaxation of purifying selection. Additionally, a few codons were found to have evidence of positive selection in mammalian RXFP3 and teleost $r x f p 4$ (stronger evidence). Although mammalian RXFP4 also showed evidence of positive selection (model 8 was preferred over models 7 and 8a); no specific codons had a BEB probability of being under strong positive selection. Overall, this suggests similar patterns of selection on ligand-receptor pairs, with the notable exception of RLN-RXFP1 in mammals for which strong evidence of positive selection exists for the ligand, but no strong evidence of positive selection on the mammalian receptor gene, $R X F P 1$.

(2b) Evidence for Differential Selection on Teleost Versus Mammalian Lineages for Orthologous Receptors. Although the above analyses suggested that only mammalian RLN has experienced high levels of codon-specific positive selection, using the branch-site model of codon-specific positive selection, we tested whether mammalian and teleost lineages have been subject to lineage-specific positive selection, that is whether they have been selected to be fixed for different amino acids (Table S5). This analysis revealed considerable evidence of lineage-specific selection indicating that mammalian and teleost lineages have evolved in different ways, and it also highlighted some important differences in the regions of the receptors that have been subject to 




(a)

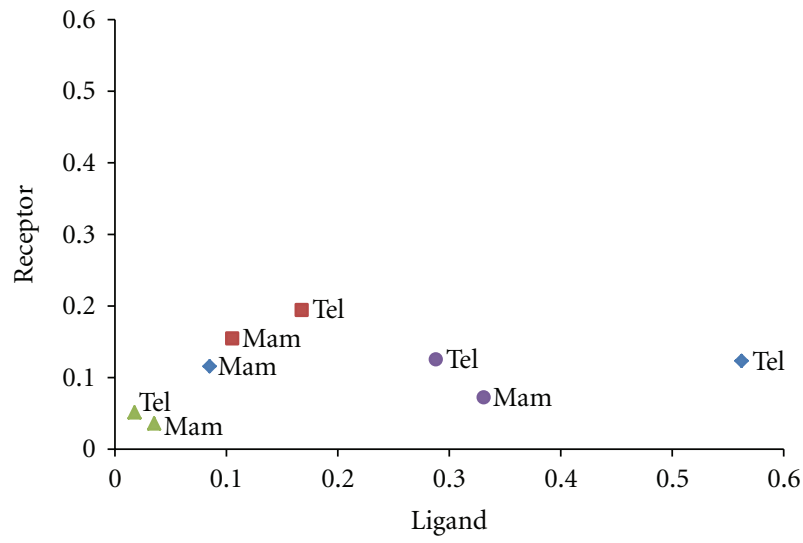

(b)



(c)

FIGURE 5: Estimated proportion of sites in the ligand ( $x$-axis) and receptor ( $y$-axis) genes evolving under (a) purifying, (b) neutral, and (c) positive selection in the genes of the putative ligand-receptor pairs of the RLN/INSL-RXFP system in mammals and teleosts.

positive selection. By mapping, codons were found to have evidence of positive selection to their position in the mature proteins; we find that (1) the low-density lipoprotein/leucine rich repeat (LDL/LRR) region of RXFP1/2-type genes is an important region of diversification among lineages; (2) for the 7 transmembrane (7TM) region shared between the two receptor types, all regions have more selected sites in RXFP3/4- than in RXFP1/2-type genes, except extracellular loop 2 (ECL2), and (3) intracellular loops 1 (ICL1) and 3 (ICL3) have many positively selected sites for RXFP3/4 genes while ICL3 also has many amino acids selected for RXFP1/2 type genes (Figure 7).

Closer examination of the sites that were selected in mammalian versus teleost lineages revealed somewhat different regions of selection in teleosts versus mammals. For RXFP1, mammals had more selection on the first few domains of the LDLa/LRR region, while teleosts exhibit greater selection on the terminal LRR domains. Additionally, in general mammalian, RXFP1 genes were found to have more selected sites in the ICLs (ICL1 and ICL3), while teleosts exhibit more selection in the ECLs (ECL1 and ECL3)
(Figure S1). This suggests that while the overall patterns of selection are similar among mammalian and teleost putative ligand-receptor orthologs, divergent selection has operated in both lineages for all genes, and some of this selection could be associated with intra- versus extracellular signaling (Figure S1).

Quantitative Expression of All Ligand and Receptor Genes in Zebrafish across Multiple Tissues. To infer functional ligandreceptor relationships, we assessed the expression of both ligand and receptor genes in male and female zebrafish heart, intestine, gonads, muscle, gills, brain, and eyes using realtime, quantitative PCR. Overall, the fold increase of the target to housekeeping genes, especially the receptors, was similar for both sexes in all tissues (except gonad) confirming the reliability of the data (Figures S2 and S3). To allow comparison of the relative amounts of mRNAs produced per tissue, the relative mRNA expression levels were normalized to the total amount of RNA isolated per tissue (Figure 8). This revealed that for all tissues studied, the expression levels 


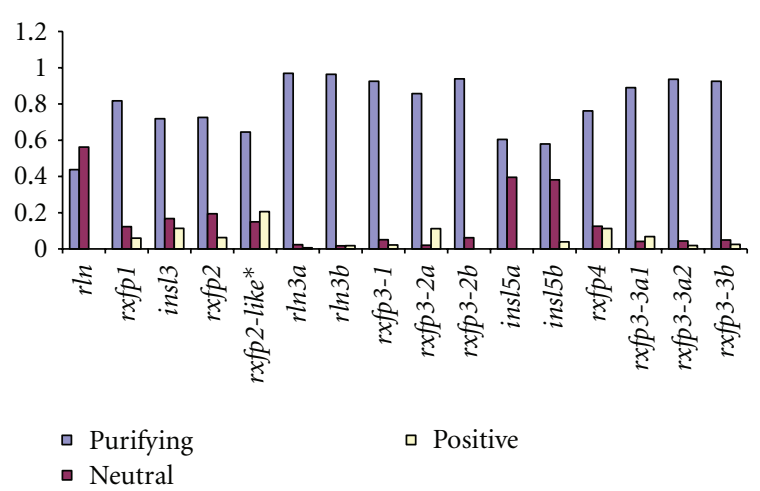

FIgURE 6: The proportion of sites in ligand and receptor genes subjected to different kinds of selection in teleosts. Selection types: purifying (light purple), neutral (dark purple), and positive (yellow). Hypothesized ligand-receptor pairs are placed in consecutive order. * Only present in zebrafish.

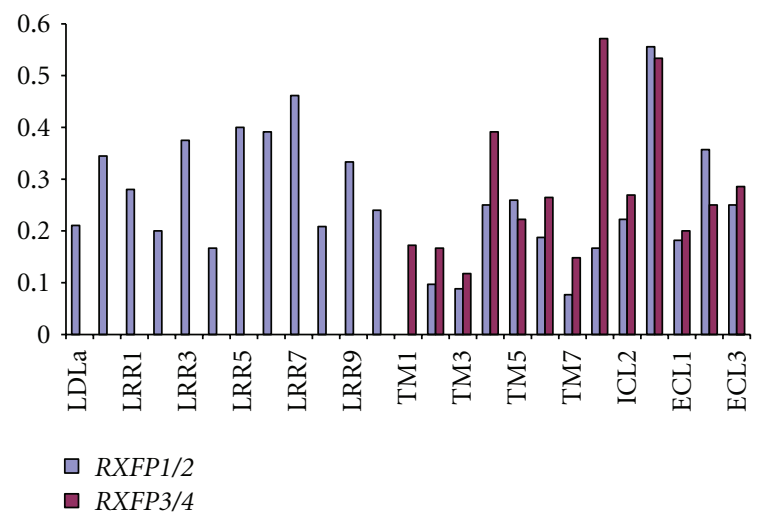

FIGURE 7: The proportion of amino acids selected per region in $R X F P 1 / 2$ and RXFP3/4 receptor genes that showed evidence of positive selection in mammalian or teleost lineages according to the branch-site test of positive selection. LDLa-low-density lipoprotein module A, LRR - leucine rich repeat, TM-transmembrane domain, ICL-intracellular loop, and ECL—extracellular loop.

of all $r x f p$ genes appeared to be higher than the expression levels of all rln/insl genes, except for the very high expression levels of insl 3 in testis tissue (Figure 8).

The ligand $r l n$ was most abundantly expressed in gonads and male intestine (Figures S2 and 8); its primary hypothesized receptor, $r x f p 1$, was also highly expressed in gonads, as was a potential secondary candidate receptor, $r x f p 2 a$ (Figure 8). The $r x f p 1$ transcript was also detected in male heart and brain, while $r x f p 2 b$ expression was found in brain and eyes. Expression of the zebrafish-specific rxfp2like transcript, a candidate receptor for Rln and Insl3, was only found in brain at high levels. Very high expression of insl3 mRNA was found in testes and somewhat lower levels in ovaries and eyes. The primary candidate receptors for Insl3 are Rxfp2a and Rxfp2b, and high expression of both $r x f p 2 a$ and $r x f p 2 b$ was observed in gonads, while $r x f p 2$-like was not detected in testes or ovaries. As expected, $r \ln 3 a$ and $r \ln 3 b$ expression was found predominantly in brain and gonad, but we also identified $r \ln 3 a$ expression in heart (Figures S2 and 8). On the other hand, all of the $\operatorname{rxpp3-}$ 1, rxfp3-2, and $r x f p 3-3$ genes showed a similar expression pattern: high expression in brain with lower levels in testes and eye, only rxfp 3-3a3 exhibited relatively low expression in brain. Relatively high levels of insl5a and insl5b mRNA were found in intestine, but additionally insl $5 a$ expression was found in gonads and brain. Our hypothesized candidate receptors for Insl5a are Rxfp3-3a1, Rxfp3-3a2, and Rxfp3-3b and for Insl5b is Rxfp3a3 (Figure 4): of the genes coding for these receptors, only $r \times f p 3-3 b$ showed high expression in the intestine (Figure 8).

\section{Discussion}

The main goal of this paper was to explore possible ligandreceptor pairings for the rln/insl-rxfp genes in teleosts. Based on previous bioinformatic analyses, we describe how teleosts preferentially retained $2 \mathrm{R}$ - and $3 \mathrm{R}$-derived paralogs of genes putatively involved in neuroendocrine functions $(r \ln 3 /$ ins $15-r x f p 3 / 4)$, ultimately leading to a greater number (10-11) of receptor genes than ligands (6). Given that the ligand-receptor pairings in teleosts are largely unknown, we employed selection and expression analyses to explore the possible ligand-receptor pairings. Overall, the selection analyses showed that (1) the extent of purifying, neutral, and positive selection acting on the four RLN-RXFP orthologs was highly similar between mammalian and teleost genes suggesting that, with the exception of mammalian RLN, ligands and receptors have the same binding relationships in both lineages and (2) the ligand-receptor pairs RLN3-RXFP3 and INSL3-RXFP2 exhibited highly similar selection profiles suggesting close coevolution, while the pair INSL5-RXFP4 exhibited a more diffuse coevolution, and RLN-RXFP1 exhibited much faster evolution of the ligand in mammals than in teleosts. The overall similarity between the genes in teleosts and mammals is supported by the observation that all of the teleost ligand genes exhibit predominant expression in the same tissues as their orthologs in mammals: rln and insl3-gonad, rln3-brain and insl5-intestine. However, even if the binding relationships are the same, it does not mean that the gene pairs have the same function in mammals and teleosts; indeed, the branch-site test of positive selection suggests that differentiation in function has occurred between the two groups. Secondly, although the binding relationships of the genes with orthologs in mammals and teleosts may be the same, it was difficult to resolve the ligand-receptor pairing relationships for the additional genes found in teleosts, but not in mammals.

\subsection{The Highly Conserved Pair RLN3-RXFP3 Expanded through Gene Duplication and Possible Subfunctionalization in Teleosts. The RLN3-RXFP3 system shows strong evidence of ligand-receptor coevolution with almost all amino acids being subject to purifying selection for both genes, and exhibiting a nearly perfect correlation in both mammals and teleosts. These findings are in accordance with previous studies and further support hypotheses about the highly}




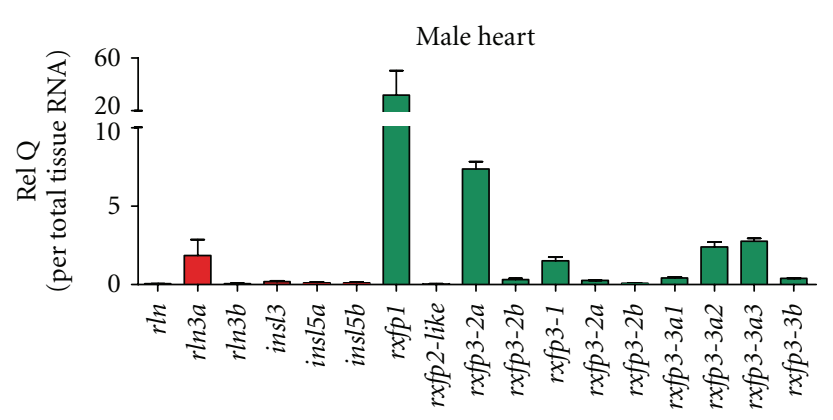

(a)

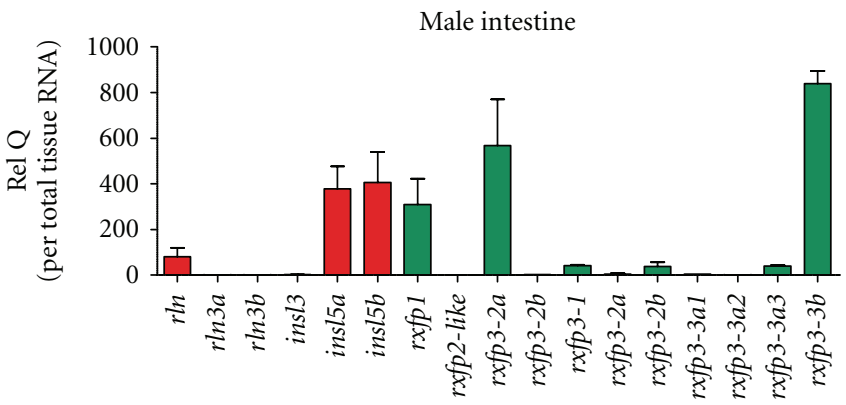

(c)

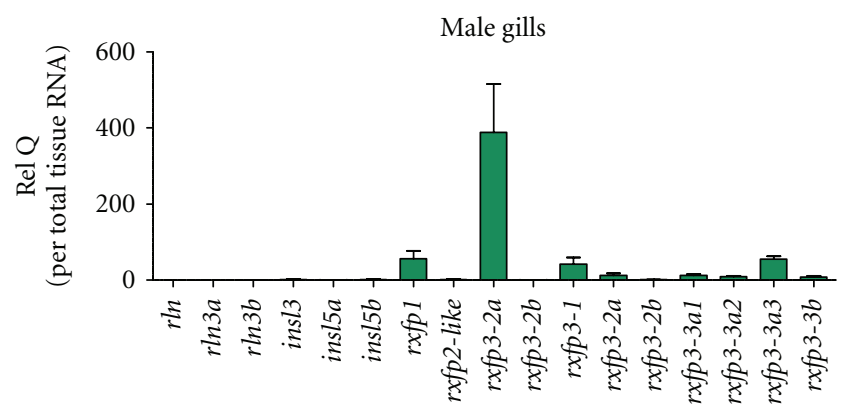

(e)

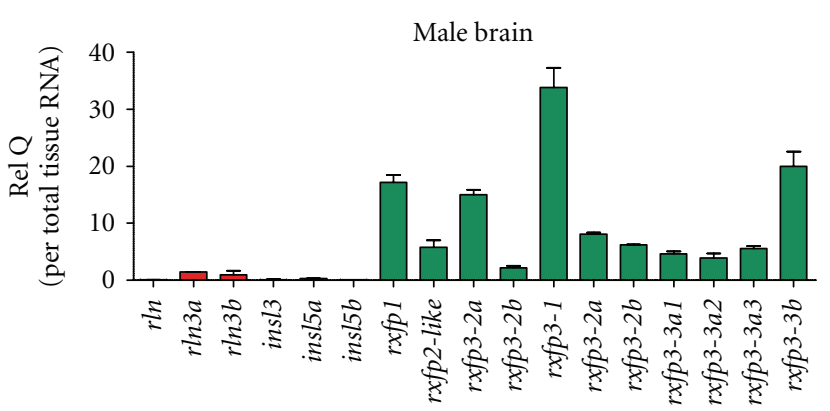

(g)

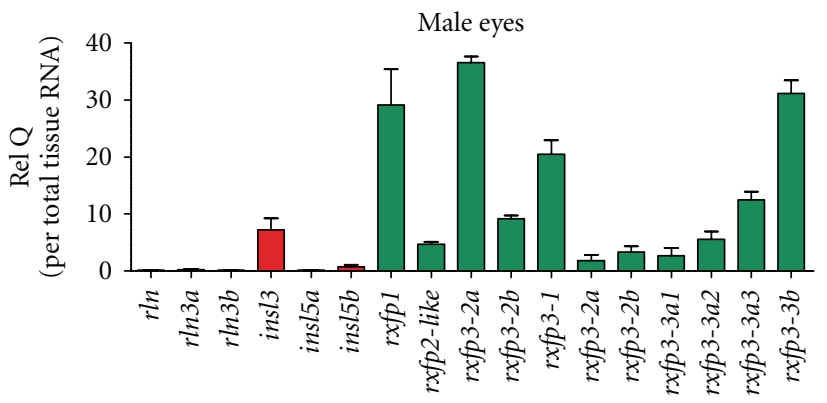

(i)

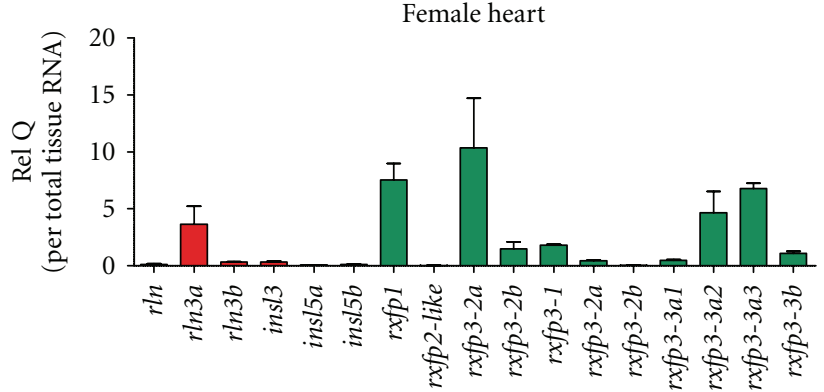

(b)

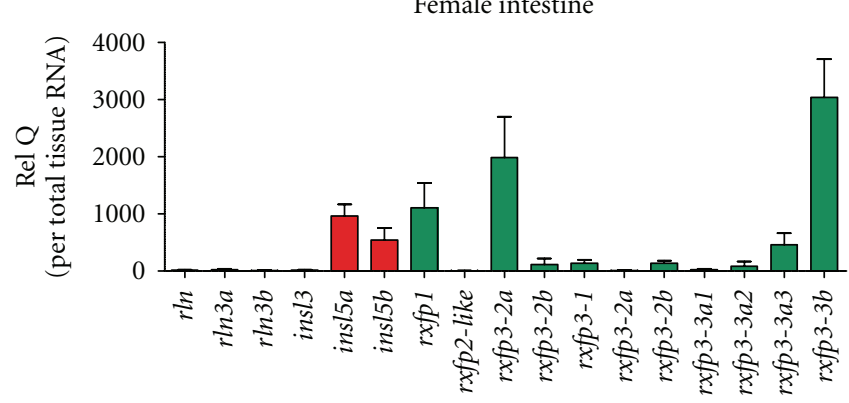

(d)

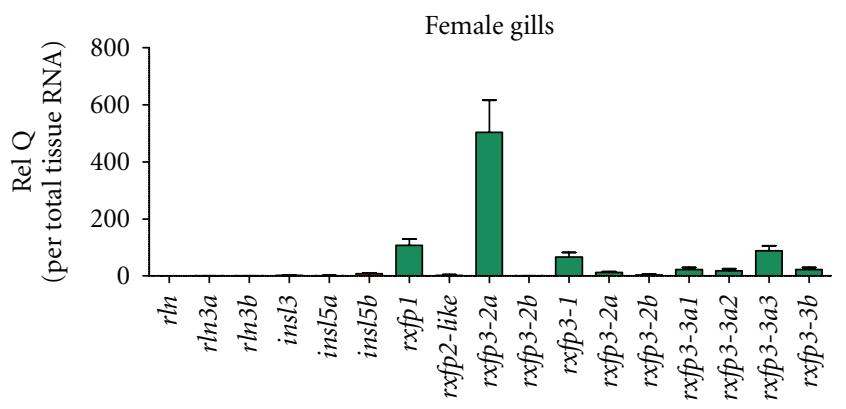

(f)

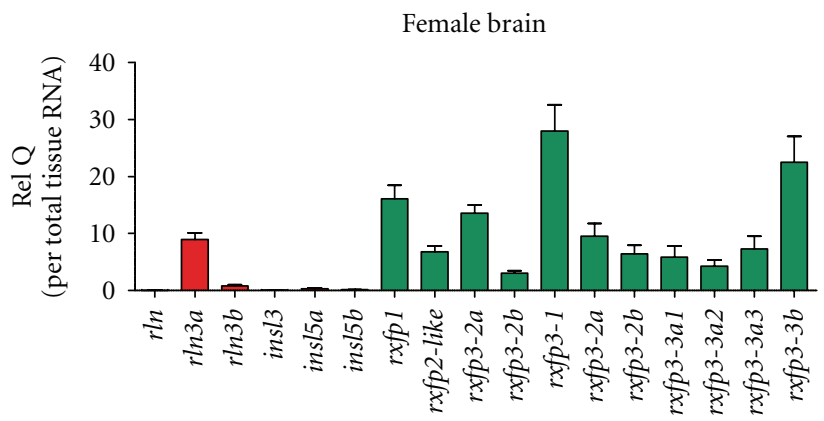

(h)

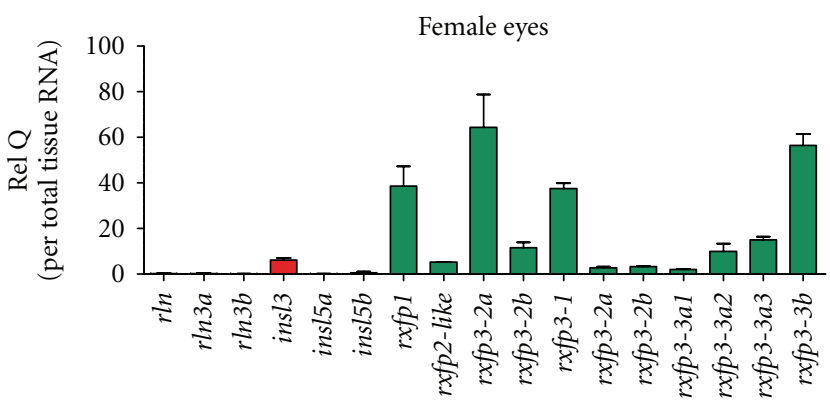

(j)

Figure 8: Continued. 


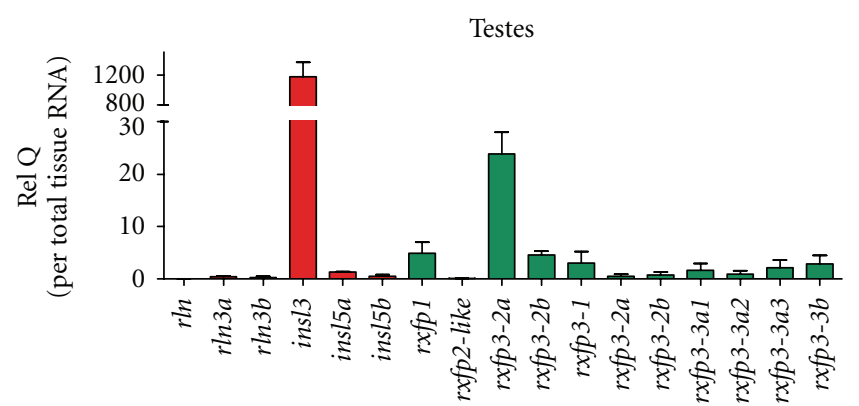

(k)

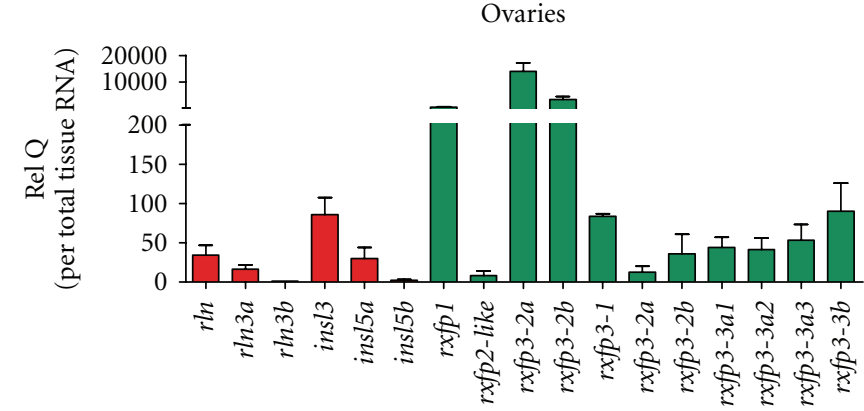

(1)

Figure 8: Relative expression of $r \ln /$ ins $l$ and $r x f p$ genes in zebrafish tissues. The expression of a gene relative to the average expression across all genes in a given tissue of males and females is shown. Red and green bars indicate the relative expression of the ligand and receptor genes, respectively. Three biological replicates were used to determine the standard errors on the relative expression.

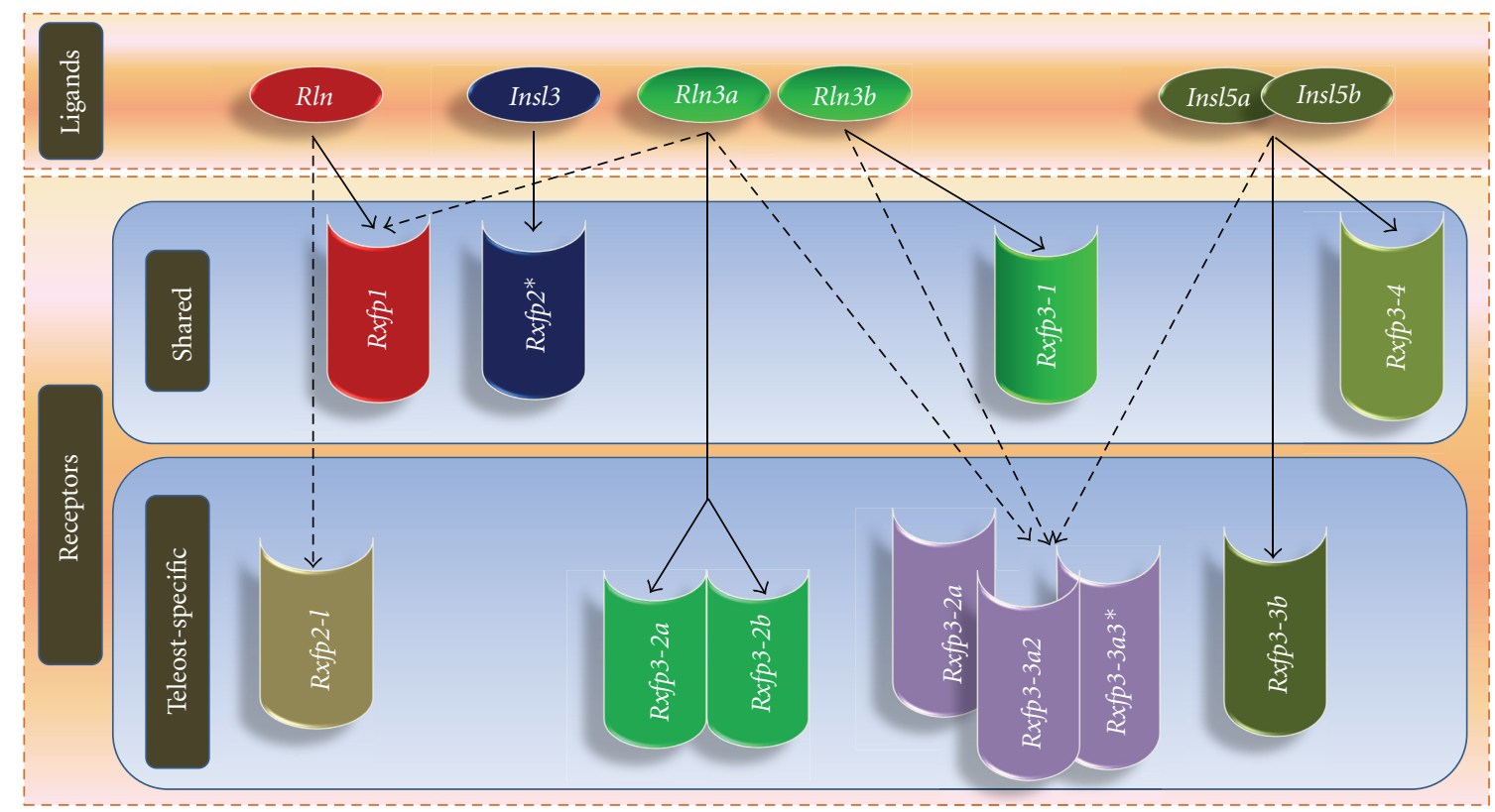

Figure 9: Proposed Rln/Insl-Rxfp ligand-receptor pairings based on previous genomic data (see Figure 4) and the analyses presented here. Solid lines represent potential ligand-receptor relationships that are well supported, while dashed lines represent uncertain pairings. There are four receptor orthologs between mammals and teleosts, and the data support the same ligand-receptor pairings for these genes in teleosts. For the seven teleost-specific receptors, strong ligand-receptor pairings were supported for three (solid lines), while the other three Rxfp3-3 receptors have unclear pairings (dotted lines), and Rxfp2-like is a probable secondary receptor for Rln.

conserved nature of the RLN3-RXFP3 genes, and their probable parallel function across most vertebrates [23]. However, teleosts possess two 3R-derived $r \ln 3$ paralogs $(r \ln 3 a$ and $r \ln 3 b)$ and multiple $r x f p 3$-type genes, not all of which are orthologous to mammalian RXFP3. Based on the duplication history of the genes [2], we proposed that the Rln3 peptide together with Rxfp3-1 and Rxfp3-2 receptors formed a tripartite ancestral teleost ligand-receptor signaling system (Figure 3), and hypothesized that the after3R subfunctionalization of the $r \ln 3$ paralogs would be associated with subfunctionalization of the $r x f p 3-1$ and $r x f p 3-2$ receptor genes (Figures 3 and 4). Taking into account that in Tetraodon nigroviridis the loss of $r \ln 3 b$ coincides with the pseudogenization of $\operatorname{rxfp} 3-1$ (Figure 3), we further propose that $R \ln 3 b$ is a cognate ligand of $R x f p 3-1$, while $R \ln 3 a$ has specialized to function with two receptors, namely, Rxfp3$2 \mathrm{a}$ and Rxfp3-2b (Figure 9). This hypothesis is supported by experimental data presented here and elsewhere. For example, experimental studies performed in zebrafish [14] and eel [9] indicate that the expression of the $r \ln 3$ paralogs in fish shows strong homology to mammalian RLN3, where they are predominantly expressed in the periaqueductal grey, a region homologous to NI in mammals. Additionally, it is known that $r \ln 3 a$ is expressed in a broader range of tissues (including gonad) than $r \ln 3 b$, indicating that $r \ln 3 a$ and $r \ln 3 b$ exhibit spatial (and perhaps temporal) subfunctionalization $[8,9,14]$. Our expression analyses indicate both coexpression of the $r \ln 3$ paralogs with $r x f p 3-1$ and $r x f p 3-2$ genes, and 
also possible subfunctionalization of the receptor since all of the $r x p p 3-1$ and $r x f p 3-2$ (and even $r x f p 3-3$ ) genes are highly expressed in brain, while $r x f p 3-2 a$ and $r x f p 3-2 b$ are additionally expressed in the ovary, but at lower levels, mimicking the expression pattern of its candidate ligand, $r \ln 3 a$.

3.2. Receptors for the Insl5 Paralogs in Teleosts Are Difficult to Resolve. Resolving the ligand-receptor pairings for the Insl5Rxfp4 system in teleosts is more difficult. We hypothesized that the Rxfp3-3 and Rxfp3-4 descendents (Figure 1) are the potential receptors for Insl5a and Insl5b (Figure 2, supplementary Figure S3). Specifically, we hypothesized that, in teleosts, Rxfp3-3a1, Rxfp3-3a2, and Rxfp3-3b are candidate receptors for Insl5a while Rxfp3-4 (aka Rxfp4) is the receptor for Insl5b; in zebrafish, the loss of $r x f p 3-4$ was compensated by the gain of $r x f p 3-3 a 3$ (Figure 2), and the latter could serve as the receptor for Insl5b (Figures 3 and 4). Despite this prediction, the selection and expression data provided little evidence for which receptors may bind to the two teleost insl5 paralogs (Figure 9). The selection profile of teleost $r \times f p 4$ is the best match for that of both insl5a and insl5b, but all three rxfp3-3-type receptors are dominated by purifying selection and have selection profiles similar to those of $r \ln 3$. On the other hand, the experimental data in zebrafish (which lacks $r \times f p 4$ ) indicate that insl5a is expressed in intestine and gonads and insl5b is expressed predominantly in intestine, and both paralogs exhibit low but significant expression in brain. This is consistent with the pattern in mammals, but the only receptor expressed at high levels in intestine was $r x f p 3-3 b$. The failure to find stronger evidence of coexpression of additional receptors for the Insl5 paralogs may be caused, in part, by the endocrine action of Insl5 and its expression in peripheral tissues $[18,24]$, many of which were not examined here, or possibly by developmental regulation of one or both of the insl5 paralogs. Three of the other Rxfp3-3 receptor genes, $r \times f p 3-3 a 1, r x f p 3-3 a 2$, and $r x f p 3-3 a 3$, were all additionally expressed in brain and male gonads, therefore if Insl5a is a ligand for these receptors, teleosts may have expanded and subfunctionalized the role of the Insl5 peptides involved in the HPG axis. Further experimental work, including in situ hybridization, should be performed on insl5 and rxfp33 receptors in teleosts to thoroughly assess this hypothesis. Furthermore, the coexpression of insl5- and rxfp3/4-type genes in a teleost species other than zebrafish should be performed since zebrafish possesses a slightly unique suite of genes (Table S2), which did not allow for qPCR analyses of rxfp4.

3.3. The INSL3-RXFP2 System Exhibits Similar Expression Patterns in Mammals and Zebrafish. While teleosts exhibit a clear expansion of the $r l n / i n s l$ and $r x f p$ genes involved in neuroendocrine pathways, the $3 \mathrm{R}$ duplicates of $r \ln$ and insl3 and their corresponding rxfp1/2-type receptors expanded minimally. We find good support for the hypothesis that Insl3-Rxfp2 are ligand-receptor pairs in teleosts: their selection profiles are highly similar and, in zebrafish, which contain two $r x f p 2$ paralogs ( $r x p p 2 a$ and $r x f p 2 b$ ), both receptor genes are highly expressed in gonads, although $r x f p 2 b$ is additionally quite highly expressed in brain. Previously, it was shown that insl3 expression in zebrafish shows strong parallels to that in mammals: in situ and qPCR analyses on male gonads reveal that it is expressed predominantly in Leydig cells [8], and the more thorough qPCR analyses presented here further demonstrate that it is very abundantly expressed in male gonads, but also in female ovaries. Current in situ analysis (underway in our laboratory) has also revealed the specificity of $r x f p 2 a$ and $r x f p 2 b$ expression in Leydig cells (unpublished data). On the other hand, although rxfp2-like (which among teleosts is only present in zebrafish) has a similar selection profile to insl3, we found it to be predominantly expressed in brain, rendering interpretation difficulty, and we favor the hypothesis that Rxfp2-like is an alternate receptor for Rln (see Figures 4 and 9).

3.4. RLN-RXFP1 System in Placental Mammals and Teleosts: Conserved Receptor but Rapidly Evolving Ligand in Mammals. The only ligand-receptor pair for which there was a poor correlation in the nature of selection was RLN-RXFP1 in mammals. While RXFP1 genes in mammals and teleosts have evolved in similar ways, the gene coding for the hormone relaxin, $r l n$, has been subject to purifying and neutral evolution in teleosts, but has been the target of strong positive selection in mammals (see Figure 5(c), Table S3). In accordance with two recent studies showing the strong role of selection on the relaxin locus $[25,26]$, we find that approximately $50 \%$ of the codons in mammalian $R L N$ show evidence of positive selection, whereas no sites in teleost $r h$ do. Additionally, the qPCR expression pattern of $r x f p 1$ in zebrafish shows broad but low levels of expression across multiple tissues, including gonad and brain. Using RT-PCR and in situ analyses in zebrafish, Donizetti et al. [27] showed that expression of $r x f p 1$ in zebrafish brain begins early in development and shows strong overlap with that of RXFP1 in humans. Based on the similar amino acid sequence of Rln and Rln3 in teleosts, they propose that Rxfp1 could be an additional receptor for $\mathrm{R} \ln 3 \mathrm{a}$ and/or $\mathrm{R} \ln 3 \mathrm{~b}$ in teleosts. A study comparing the expression of $r \ln 3 a, r \ln 3 b$, and $r \ln$ in eel using in situ and qPCR analyses [9] found that the expression of teleost $r \ln$ is similar to that of $r \ln 3$, but with lower expression in brain and higher in gonads, similar to that observed in which expression was predominantly found in gonad. This pattern is supported by our hypothesis for the evolution of the system in which the ancestral ligand molecule is hypothesized to have functioned in both reproductive and neuroendocrine pathways (Figure 3).

\subsection{Evidence for Differential Selection in Teleost Versus Mam- malian rln/insl-rxfp Genes Suggests Functional Divergence of the Ligand-Receptor Coding Sequences. Although we have focused on the similarities in the evolution of mammalian and teleost RLN/INSL-RXFP genes, the analysis of codon- specific positive selection revealed that mammalian and teleost genes have been subject to differential selection and that some receptor domains are the targets of more selection}


than others. For this analysis, sites were deemed to be subject to codon-specific selection if, when comparing a particular branch of the phylogenetic tree, there was evidence that certain amino acids were selected to be different from those in the "background" lineage for the same gene. By analyzing the genes in this way, we found that for the RXFP1/2-type genes, the LDLa-LRR region generally showed high levels of selection, not surprisingly, since they are involved in receptor-ligand signaling [5]. Functional studies have shown that the LRR region is important for the binding of the cognate ligand; the LDLa module is essential for cAMP accumulation which takes place after the ligand is recognized and bound [5]. Apart from these regions, the only other two regions which were identified as having more than $20 \%$ of the sites subject to selection for $R X F P 1 / 2$ genes were ICL3 and ECL2.

In general, lineage-specific selection was higher for the RXFP3/4-type genes: all domains were found to have more than $20 \%$ of the amino acids subject to positive selection except for four regions of the transmembrane domain (TM1, TM2, TM3, andTM7) and ECL1. Of particular interest is the fact that for the RXFP3/4-type genes, ICL1 is equally important as ICL3 in terms of selection. The finding that ICL3 (both receptor types) and ICL1 (RXFP3/4-type receptors) are targets of selection suggests that a major component of selection for the RXFP receptors concerns downstream receptor signaling rather than selection for ligand binding per se.

\section{Conclusions}

Although the majority of the relaxin family genes originated prior to the divergence of osteichthyans, the fate of the family in teleosts and mammals is markedly different owing to the differential retention and diversification of genes in each lineage. Earlier studies suggested that teleosts only possessed relaxin 3- and rxfp3-like genes and proposed that RLN and INSL3 were neurohormones that recruited their RXFP1/2type receptors after the divergence of mammals [28], a view that is inconsistent with the data presented here and elsewhere $[2,8,29,30]$. The goal of this study was to establish a theoretical background for further experimental work on the rln/insl-rxpf systems in teleosts. Although the study was limited because its methodology relied on the known ligand-receptor pairings and expression data from mammals as a reference, our analyses suggest that the orthologs of the four 2R-derived ligand genes ( RLN, INSL3, RLN3, and INSL5) have similar ligand-receptor pairings in teleosts and mammals (with the exception of the unusual situation with Rln-Rxfp1). Despite these similar patterns, there is also evidence of differential selection on specific amino acids in mammalian versus teleost lineages, suggesting functional divergence in the two lineages.

It is interesting that the RLN/INSL peptides diversified their reproductive functions in mammals, owing to local duplications at the relaxin locus [23, 25, 26, 29], while teleosts underwent a massive diversification of the genes believed to be involved in neuroendocrine regulation
( $r \ln 3 /$ insl5-rxpf3/4). Overall, we find evidence that many of these "additional" receptor genes in teleosts have characteristics of the RLN3-RXFP3 system, that is, slow evolution and predominant expression in the brain, while the primary receptors for the two Insl5 paralogs in teleosts remain obscure. Nevertheless, we find that teleosts greatly expanded and probably subfunctionalized the role of the rxfp3-2and rxfp3-3-derived receptors; their cognate ligands and their physiological functions should be the focus of future experimental work.

\section{Materials and Methods}

5.1. Selection Profiles of Candidate Ligand-Receptor Pairs. We obtained sequences and performed an alignment based on the coding sequence for the RLN/INSL-RXFP genes from 5 teleosts (zebrafish, medaka, fugu, tetraodon, and stickleback) and 11 placental mammals (human, rhesus, cow, pig, horse, dog, guinea pig, mouse, rat, rabbit, and elephant) as described previously [2]. The accession numbers of all genes are listed in Tables S4 and S7 in Yegorov and Good [2], and the alignment is available upon request.

We calculated the proportion of codons in ligand and receptor pairs estimated to be subject to purifying, neutral, or positive selection using the sites model in PAML [22]. Next, to assess whether teleost ligand or receptor genes have been subject to adaptive divergent selection, we used several methods that examine the ratio of nonsynonymous to synonymous $\left(d_{N} / d_{S}\right)$ substitutions. Because $d_{S}$ provides an approximation of the neutral rate of substitution, $\omega=d_{N} / d_{S}$ ratios are used to determine selection pressure on genes or codon positions, with $\omega>1$ indicative of positive Darwinian selection [31].

Site Models. We employed models that allow $\omega$ to vary among sites and tested a series of models to look for evidence of positive selection. First, we compared model M7 (beta) versus M8 (beta $+\omega$ ) to test for evidence of positive selection and then compared model 8 versus model $8 \mathrm{a}$ to assess whether the evidence for positive selection was actually caused by a relaxation of purifying selection (or true positive selection); for both comparisons we used the site model tests in PAML [32]. Likelihood ratio tests (LRTs) were constructed to compare model M7 versus M8 and M8a versus M8. Twice the log likelihood difference between models was compared with a chi-square distribution with number of degrees of freedom $(d f)$ calculated as the difference in the number of estimated parameters between models. Model M8 was additionally used to identify codon sites under positive selection using a Bayes Empirical Bayes (BEB) criterion.

Branch-Site Models. We hypothesized that at least some of the receptor genes may have experienced lineage-specific positive selection in mammals versus teleosts. To examine this we used the branch-site model A of Zhang et al. [22], which tests whether the members of a user-defined clade (branch) on a phylogenetic tree exhibit evidence of codonspecific selection relative to the remaining (background) 
lineages. Tests of positive selection were made by comparing the branch-site model $\mathrm{A}$ in which $\left(d_{N} / d_{S}\right)>1$ (alternative hypothesis) to the model $\mathrm{A}$ in which $d_{N} / d_{S}=1$ fixed (null hypothesis) and by setting the foreground branch to the base of the clade containing the relaxin family ortholog in teleosts and the background to the same ortholog in mammals or tetrapods (depending on the tree structure) or vice versa. Analysis of the branch-site model A was done using CODEML from the PAML package (PAML v. 4.2); models were compared using the likelihood ratio test with 1 degree of freedom and, where significant, the posterior probability that a codon was under positive selection was estimated using the Bayes Empirical Bayes (BEB) procedure [22].

\subsection{Quantitative Expression Analysis in Zebrafish Tissues} Animals. Sexually mature male and female zebrafish (Danio rerio) from the Tübingen $\mathrm{AB}$ strain were used. Animal housing [33] and experimentation were consistent with Dutch national regulations and were approved by the Utrecht University Animal Use and Care Committee.

RNA Isolation and cDNA Synthesis. Various tissues (heart, intestine, testis, ovary, muscle, gill, brain, and eye) were dissected from male and female adult zebrafish and immediately flash frozen in liquid nitrogen.Tissue samples from 3 individual zebrafish, for each gender, were combined for each replicate and the RNA was isolated using the FastRNA Pro Green kit (Bio 101 Systems), according to the manufacturer's recommendations. Three independent RNA isolations (biological replicates), each containing pooled tissues from 3 individual fish, were performed for each tissue per sex. Possible genomic DNA contamination was removed from each total RNA fraction with the RNAse-free DNase Treatment \& Removal kit (Ambion), which includes a final step to remove the DNAse I from the reaction. Next, cDNA synthesis was performed with $2 \mu \mathrm{g}$ of each total RNA samples, as described previously [34].

Real-Time, Quantitative PCR. Primers (Table S6) for realtime, quantitative PCR ( $\mathrm{PPCR}$ ) to detect zebrafish $\mathrm{rln} / \mathrm{ins}$ l and $r x f p$ mRNAs were designed and validated for specificity and amplification efficiency on serial dilutions of testis cDNA [35] using SYBR Green-based assays (Applied Biosystems, Foster City, CA, USA). All primers were designed on different exons, except for the primers detecting the rxfp3 cDNAs, since all $r x f p 3$ genes are single-exon genes. Moreover, each qPCR run was followed by a melt curve analyses to exclude potential PCR amplifications from genomic DNA contamination. To normalize the data, a TaqMan Gene Expression Assay was acquired to detect the endogenous control RNA, eukaryotic $18 \mathrm{~S}$ ribosomal RNA (Applied Biosystems). To examine the relative expression of genes across tissues, the relative fold change of the genes of interest was normalized to the $18 S$ ribosomal $R N A$ reference gene and to a calibrator (calculated as the mean expression of all genes) (supplementary Figures S2 (ligands) and S3 (receptors). All qPCRs and calculations (using the $\Delta \Delta \mathrm{C}_{T}$ method) were performed as described previously [35-37]. To compare the expression levels of all relaxin family peptide and receptor genes in whole zebrafish tissues, expression levels were additionally corrected for the total RNA yield per tissue per sex (Figure 8).

\section{Appendix}

See Supplementary Tables S1, S2, S3, S4, S5, and S6 and Figures S1, S2, and S3 (see Supplementary materials available online at doi:10.1155/2012/310278).

\section{Acknowledgments}

The authors thank Murray Wiegand and Kevin Campbell for comments on an earlier version of this work. The authors also thank two anonymous reviewers whose comments improved this paper. Parts of this research were included in the M.S. theses of S. Yegorov and J. Martijn. This research was funded by a discovery grant from the National Science and Engineering Research Council (NSERC) to S. Good and by a Manitoba Graduate Scholarship and University of Winnipeg Graduate Award to S. Yegorov.

\section{References}

[1] O. D. Sherwood, "Relaxin's physiological roles and other diverse actions," Endocrine Reviews, vol. 25, no. 2, pp. 205-234, 2004.

[2] S. Yegorov and S. Good, "Using paleogenomics to study the evolution of gene families: origin and duplication history of the relaxin family hormones and their receptors," PLoS ONE, vol. 7, no. 3, Article ID e32923, 2012.

[3] M. L. Halls, R. A. D. Bathgate, and R. J. Summers, "Relaxin family peptide receptors RXFP1 and RXFP2 modulate cAMP signaling by distinct mechanisms," Molecular Pharmacology, vol. 70, no. 1, pp. 214-226, 2006.

[4] M. Kinoshita, K. Murata, K. Naruse et al., Medaka: Biology, Management, and Experimental 1 Protocols, Wiley-Blackwell, Iowa City, IA, Iowa, USA, 2009.

[5] M. L. Halls, E. T. Van Der Westhuizen, R. A. D. Bathgate, and R. J. Summers, "Relaxin family peptide receptors - Former orphans reunite with their parent ligands to activate multiple signalling pathways," British Journal of Pharmacology, vol. 150, no. 6, pp. 677-691, 2007.

[6] R. C. K. Kong, P. J. Shilling, D. K. Lobb, P. R. Gooley, and R. A. D. Bathgate, "Membrane receptors: structure and function of the relaxin family peptide receptors," Molecular and Cellular Endocrinology, vol. 320, no. 1-2, pp. 1-15, 2010.

[7] S. Y. Hsu, K. Nakabayashi, S. Nishi et al., "Activation of orphan receptors by the hormone relaxin," Science, vol. 295, no. 5555, pp. 671-674, 2002.

[8] S. V. Good-Avila, S. Yegorov, S. Harron et al., "Relaxin gene family in teleosts: phylogeny, syntenic mapping, selective constraint, and expression analysis," BMC Evolutionary Biology, vol. 9, no. 1, article 293, 2009.

[9] G. B. Hu, M. Kusakabe, and Y. Takei, "Localization of diversified relaxin gene transcripts in the brain of eels," General and Comparative Endocrinology, vol. 172, no. 3, pp. 430-439, 2011. 
[10] K. Kawamura, J. Kumagai, S. Sudo et al., "Paracrine regulation of mammalian oocyte maturation and male germ cell survival," Proceedings of the National Academy of Sciences of the United States of America, vol. 101, no. 19, pp. 7323-7328, 2004.

[11] S. L. Dun, E. Brailoiu, Y. Wang et al., "Insulin-like peptide 5: expression in the mouse brain and mobilization of calcium," Endocrinology, vol. 147, no. 7, pp. 3243-3248, 2006.

[12] B. M. McGowan, S. A. Stanley, J. Donovan et al., "Relaxin-3 stimulates the hypothalamic-pituitary-gonadal axis," American Journal of Physiology, vol. 295, no. 2, pp. E278-E286, 2008.

[13] C. M. Smith, P. J. Shen, A. Banerjee et al., "Distribution of relaxin-3 and RXFP3 within arousal, stress, affective, and cognitive circuits of mouse brain," Journal of Comparative Neurology, vol. 518, no. 19, pp. 4016-4045, 2010.

[14] A. Donizetti, M. Fiengo, S. Minucci, and F. Aniello, "Duplicated zebrafish relaxin-3 gene shows a different expression pattern from that of the co-orthologue gene," Development Growth and Differentiation, vol. 51, no. 8, pp. 715-722, 2009.

[15] Y. Watanabe, Y. Miyamoto, T. Matsuda, and M. Tanaka, "Relaxin-3/INSL7 regulates the stress-response system in the rat hypothalamus," Journal of Molecular Neuroscience, vol. 43, no. 2, pp. 169-174, 2011.

[16] D. Conklin, C. E. Lofton-Day, B. A. Haldeman et al., "Identification of INSL5, a new member of the insulin superfamily," Genomics, vol. 60, no. 1, pp. 50-56, 1999.

[17] S. Y. Hsu, "Cloning of two novel mammalian paralogs of relaxin/insulin family proteins and their expression in testis and kidney," Molecular Endocrinology, vol. 13, no. 12, pp. 21632174, 1999.

[18] C. Liu, C. Kuei, S. Sutton et al., "INSL5 is a high affinity specific agonist for GPCR142 (GPR100)," Journal of Biological Chemistry, vol. 280, no. 1, pp. 292-300, 2005.

[19] M. L. Halls, C. P. Bond, S. Sudo et al., "Multiple binding sites revealed by interaction of relaxin family peptides with native and chimeric relaxin family peptide receptors 1 and 2 (LGR7 and LGR8)," Journal of Pharmacology and Experimental Therapeutics, vol. 313, no. 2, pp. 677-687, 2005.

[20] D. Cyranoski, “Two by two," Nature, vol. 458, no. 7240, pp. 826 $-829,2009$.

[21] A. B. Prasad, M. W. Allard, and E. D. Green, "Confirming the phylogeny of mammals by use of large comparative sequence data sets," Molecular Biology and Evolution, vol. 25, no. 9, pp. 1795-1808, 2008.

[22] J. Zhang, R. Nielsen, and Z. Yang, "Evaluation of an improved branch-site likelihood method for detecting positive selection at the molecular level," Molecular Biology and Evolution, vol. 22, no. 12, pp. 2472-2479, 2005.

[23] T. N. Wilkinson, T. P. Speed, G. W. Tregear et al., "Evolution of the relaxin-like peptide family," BMC Evolutionary Biology, vol. 5, article 14, 2005.

[24] C. Liu and T. W. Lovenberg, "Relaxin-3, INSL5, and their receptors," Results and Problems in Cell Differentiation, vol. 46, pp. 213-237, 2008.

[25] J. I. Arroyo, F. G. Hoffman, and J. C. Opazo, "Gene duplication and positive selection explains unusual physiological roles of the relaxin gene in the European rabbit," Journal of Molecular Evolution, vol. 74, no. 1-2, pp. 52-60, 2012.

[26] J. I. Arroyo, F. G. Hoffmann, and J. C. Opazo, "Gene turnover and differential retention in the relaxin/insulin-like gene family in primates," Molecular Phylogenetics and Evolution, vol. 63, no. 3, pp. 768-776, 2012.

[27] A. Donizetti, M. Fiengo, R. Del Gaudio, R. Di Giaimo, S. Minucci, and F. Aniello, "Characterization and developmental expression pattern of the relaxin receptor $\operatorname{rxfp} 1$ gene in zebrafish," Development Growth and Differentiation, vol. 52, no. 9, pp. 799-806, 2010.

[28] R. Ivell, M. Kotula-Balak, D. Glynn, K. Heng, and R. AnandIvell, "Relaxin family peptides in the male reproductive system-a critical appraisal," Molecular Human Reproduction, vol. 17, no. 2, pp. 71-84, 2011.

[29] F. G. Hoffmann and J. C. Opazo, "Evolution of the relaxin/insulin-like gene family in placental mammals: implications for its early evolution," Journal of Molecular Evolution, vol. 72, no. 1, pp. 72-79, 2011.

[30] J. I. Park, J. Semyonov, L. C. Chia, W. Yi, W. Warren, and S. Y. T. Hsu, "Origin of INSL3-mediated testicular descent in therian mammals," Genome Research, vol. 18, no. 6, pp. 974985,2008

[31] Z. Yang and J. P. Bielawski, "Statistical methods for detecting molecular adaptation," Trends in Ecology \& Evolution, vol. 15, no. 12, pp. 496-503, 2000.

[32] R. Nielsen and Z. Yang, "Likelihood models for detecting positively selected amino acid sites and applications to the HIV-1 envelope gene," Genetics, vol. 148, no. 3, pp. 929-936, 1998.

[33] M. Westerfield, The Zebrafish Book: A Guide for the Laboratory Use of Zebrafish (Danio rerio), University of Oregon Press, Eugene, Ore, USA, 2000.

[34] F. E. M. Rebers, C. P. Tensen, R. W. Schulz, H. J. T. Goos, and J. Bogerd, "Modulation of glycoprotein hormone $\alpha$ - and gonadotropin IIß-subunit mRNA levels in the pituitary gland of mature male African catfish, Clarias gariepinus," Fish Physiology and Biochemistry, vol. 17, no. 1-6, pp. 99-108, 1997.

[35] J. Bogerd, M. Blomenröhr, E. Andersson et al., "Discrepancy between molecular structure and ligand selectivity of a testicular follicle-stimulating hormone receptor of the African catfish (Clarias gariepinus)," Biology of Reproduction, vol. 64, no. 6 , pp. $1633-1643$.

[36] P. P. de Waal, D. S. Wang, W. A. Nijenhuis, R. W. Schulz, and J. Bogerd, "Functional characterization and expression analysis of the androgen receptor in zebrafish (Danio rerio) testis," Reproduction, vol. 136, no. 2, pp. 225-234, 2008.

[37] Á. García-López, J. Bogerd, J. C. M. Granneman et al., "Leydig cells express follicle-stimulating hormone receptors in African catfish," Endocrinology, vol. 150, no. 1, pp. 357-365, 2009. 



Submit your manuscripts at

http://www.hindawi.com
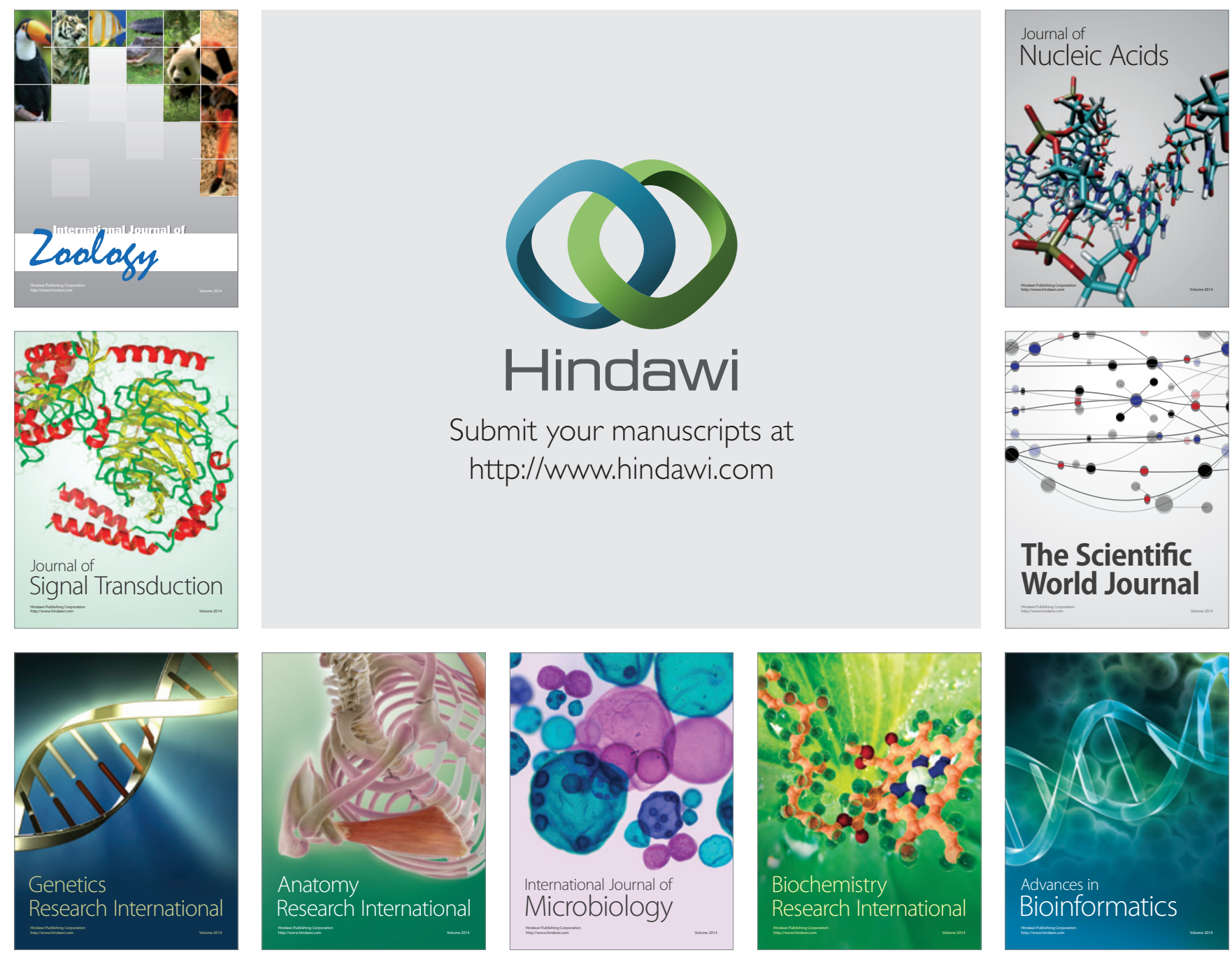

The Scientific World Journal
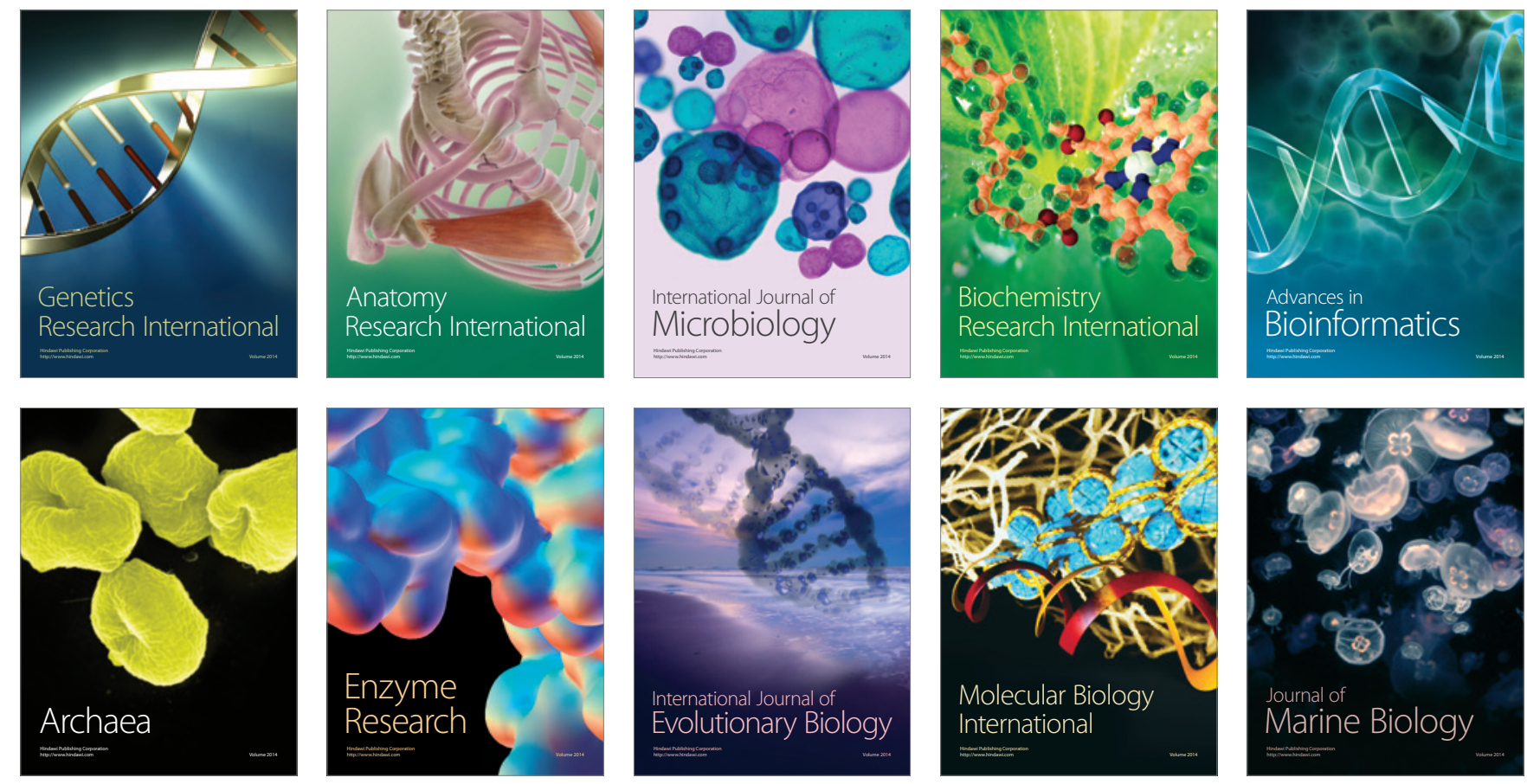